\title{
Triplet Paradox in Special Relativity and Discrepancy with Electromagnetism
}

\author{
Vladimir Alexandr Leus \\ Sobolev Institute of Mathematics, Novosibirsk, Russia
}

Email address:

vladalex@liv.ac.uk

\section{To cite this article:}

Vladimir Alexandr Leus. Triplet Paradox in Special Relativity and Discrepancy with Electromagnetism. American Journal of Modern Physics. Special Issue: Physics of Time: Theory and Experiment. Vol. 4, No. 2-1, 2015, pp. 26-33. doi: 10.11648/j.ajmp.s.2015040201.15

\begin{abstract}
Special Relativity Theory (SRT) is considered to be compatible with classical electrodynamics. However, there are several logically deduced discrepancies inherent in SRT itself, which are called "paradoxes" and demand scrupulous examination. The "twin paradox" is most well-known. In this paper a new thought experiment, including three different observers (triplets) is presented. As opposed to the notorious "twin paradox" we suggest a case where two persons are moving in opposite directions in a manner symmetrical to the basic frame of reference being fixed. This consideration leads to the fundamental notion of physics of time controlling causality as an indispensable element of every scientific view. This is a pivotal tenet of SRT - "relativity of simultaneity" that is subjected to violation, in which case the ability of lawful implementation for Lorentz transform gets in doubt. Moreover, the consistent implementation of SRT in electromagnetism leads to breakdown of the equivalence principle in the realm of General Relativity.
\end{abstract}

Keywords: Special Relativity Theory, Relativity of Simultaneity, Paradoxes, Electromagnetism, Equivalence Principle

\section{Introduction}

The 'twin paradox' - a scientific thought experiment - is the most well-known generally because of the discussion about a visit to a neighbouring star and the return back in the course of a human lifetime. Briefly summarised it is as follows: there are two persons of the same age (identical twins). One of them remains on the Earth, whereas the other travels far to space and returns later. When they meet, the stay-at-home twin appears to be older than traveller. The higher the speed and duration of the journey, the bigger is the apparent age difference. An explanation for the discrepancy has been suggested by special relativity theory (SRT) and, moreover, by several methods [1, pp. 155-156]. In SRT the divergence the ages is said to occur due to the inevitable dynamic asymmetry: the astronaut twin experiences an acceleration-deceleration event but his stationary twin brother is remaining at all times in the same inertial frame. According to French: 'There is no paradox, and the asymmetrical ageing is real' [1, p. 156], to which many other authors concur.

S. Boblest et al examined an extension of the problem to curved space-time in an expanding universe [2]. Although this reveals some new aspects of the situation and pointed out some limitations, the 'paradox' may be explained in the manner similar to the case of flat Minkowskian space-time [3]. The space-time interval " $s$ " between two events is a fundamental notion of SRT not just because its square is invariant, which is the same for all inertial observers (i.e. independent of the observer reference frame). But " $s$ " is also important in its role in the notion of 'relativity of simultaneity'. For instance if $s^{2}>0$ (time-like interval), the strict order of events is determined. For $s^{2}<0$ (space-like interval) a strict order of events does not exist. It should be noted a constructive critique of the base of SRT given in [4]. But it has, so to speak, an outward orientation, whereas the present paper deals with essentially internal discrepancy.

This paper is intended to give an extension of the wellknown twin paradox to include some another aspect. It suggests a further symmetrisation in the trip that reveals a real controversy at the heart of SRT [5]. So long as the excellent compatibility of SRT with classical electrodynamics is a common knowledge, it would be natural to seek some corresponding controversy in the very realm of electromagnetism itself. In the second part of the present paper such a cue will be given. 


\section{Preparation for the Journey}

Consider a vast country 'Relativia' that has an extremely even relief (no mountains). At the centre of the country is the capital city Einsteinville. At a platform of its railway station two identical locomotives $A$ and $B$ stand side by side on two parallel rectilinear tracks (figure 1). Each locomotive has a driving cabin at each end: a blue one and a red one. There are eight ideal identical clocks: two at the blue cabin and two at the red cabin of the locomotive $A$, two at the blue cabin and two at the red cabin of the locomotive $B$. All the clocks (red and blue) are in initial agreement with the station clock. One clock in each of the four cabins is fitted externally (i.e. on the outside of the locomotive facing the neighbouring one), so that the 'blue' clock of one locomotive may touch the 'blue' clock of the other locomotive. Similarly one of the 'red' clocks in both locomotives may touch its counterpart (figure $1)$.

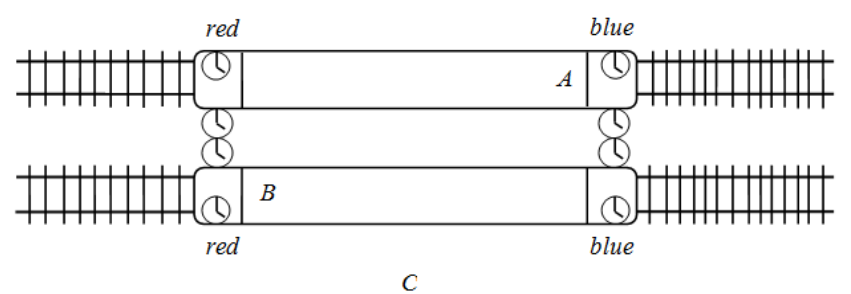

Figure 1. Starting position.

Two of three identical triplet brothers Alex and Boris are the engine-drivers on the first and on the second locomotives respectively. Both brothers make preparation of their machines for a long journey in the two opposite directions at a very high speed. The third brother Charles - being a station master is to be left at the station. Before departure he hands symmetrical exit route cards with instructions to both his brothers, and provided two sealed (tamper proof) accelerometers: one fitted on locomotive $A$, and other fitted on locomotive $B$.

\section{Crucial Experiment}

Brother Alex takes place in his blue cabin whereas Boris takes place in his red cabin. At a given moment $t_{0}=t^{\prime}{ }_{0}=$ $t^{\prime \prime}{ }_{0}$ both locomotives start a move along the rail tracks in straight lines in opposite directions. The clocks on each locomotive show the proper time for that locomotive: $t^{\prime}$ for $A$, and $t$ " for $B$. According to their instructions each brother causes his train to undertake uniformly accelerated movement with constant accelerations " $a$ " and " $-a$ " respectively until the point in time $t^{\prime}{ }_{1}=t^{\prime \prime}{ }_{1}$. At this instant they stop their engines and brake, changing acceleration for deceleration: " $-a$ " and " $a$ " respectively (figure 2). The trains stop at $t_{2}^{\prime}=t^{\prime \prime}{ }_{2}$. Each brother immediately causes his locomotive in move backwards with constant acceleration: $"-a$ " and " $a$ " respectively. When the speed value $v$ is reached at the point $t^{\prime}{ }_{3}=t^{\prime \prime}{ }_{3}$, the acceleration is brought to zero and the uniform movement continues past the Einsteinville station to the point in time $t^{\prime}{ }_{4}=t^{\prime \prime}{ }_{4}$.

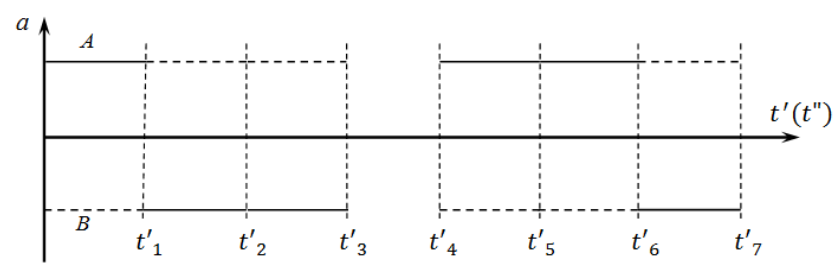

Figure 2. Planned acceleration for both locomotives (in their own reference frames)

When the locomotives pass one another, their external blue clocks touch momentarily and stop going at this point in time $t^{\prime}{ }_{b}, t^{\prime \prime}{ }_{b}$. The external red clocks also touch and similarly stop at $t_{r}^{\prime}, t^{\prime \prime}{ }_{r}$. At a later time $t^{\prime}{ }_{4}=t^{\prime \prime}{ }_{4}$ the brakes are applied providing decelerations " $a$ " for the $A$, and " $-a$ " for the $B$ until both locomotives stop at the time point $t^{\prime}{ }_{5}=t^{\prime \prime}{ }_{5}$ at the turning point. The brothers again make them move back with accelerations " $a$ " for $A$ and " $-a$ " for $B$. At the point $t^{\prime}{ }_{6}=t^{\prime \prime}{ }_{6}$ they change accelerations for the decelerations " $-a$ " for the $A$ and " $a$ " for the $B$. Finally, both locomotives stop side by side at $t^{\prime}{ }_{7}=t^{\prime \prime}{ }_{7}$ near the platform of the Einsteinville railway station. At this particular instant the station's clock shows $t_{7}$, which is the local time. This time must satisfy inequalities: $t_{7}>t^{\prime}{ }_{7}$, and $t_{7}>t^{\prime \prime}{ }_{7}$ in accordance with the well known solution of the "twin paradox'.

\section{Result of the Crucial Experiment}

Consider two events: $e_{b}$ - interaction between the blue clocks, and $e_{r}$-interaction between the red clocks when the locomotives pass one another. There exists an inertial frame where both these events occur at the same moment. That is the platform's frame of reference where events under consideration occur at points $x_{b}$ and $x_{r}$ respectively, and at a moment $t_{b r}=t_{b}=t_{r}$. Thus, $s\left(e_{b}, e_{r}\right)$ is a space-like interval and therefore the order of events appears to be indefinite. Lorentz transform gives the following expressions:

$$
t_{r}^{\prime}-t^{\prime}{ }_{b}=v \frac{\gamma}{c^{2}}\left(x_{r}-x_{b}\right) ; t^{\prime \prime}{ }_{r}-t^{\prime \prime}{ }_{b}=-v \frac{\gamma}{c^{2}}\left(x_{r}-x_{b}\right),
$$

Where $v$ is speed of a locomotive, $c$ is speed of light, and relativistic factor $\gamma=\left(1-v^{2} / c^{2}\right)^{-1 / 2}$. The right-hand sides of equations (1) have different signs because the locomotives are moving in opposite directions symmetrically to the platform.

After arrival Alex brings forward his stopped red and blue clocks:

$$
t_{r}^{\prime}<t_{b}^{\prime}
$$

Boris shows the readings of his stopped clocks:

$$
t_{b}<t_{r}^{\prime} \text {. }
$$

"This is the relativity of simultaneity.' [1, pp. 74-79] says Alex. "SRT is proven once again!" - Boris replies. "Brothers, do you believe in SRT?" - Charles asks. "Certainly!" - they answer. "So you accept the 'twin paradox' result, and you know that we now are no longer identical triplets as before. Our identical age status finished at the 
starting time of the journey. Now I am your senior and I invite you to take a look from my position at the platform. Since the moments $t^{\prime}$ and $t^{\prime \prime}{ }_{7}$ of your arrival at the Einsteinville station are equal, then $t^{\prime}{ }_{7}=t^{\prime \prime}{ }_{7}$ and so you have now identical ages. However this does not necessarily mean that your journeys have mirrored one another. To ascertain this we need to inspect the records of your accelerometers." Charles replies. The devices at both locomotives were unsealed and checked against the planned motion (figure 2). Both drivers had obeyed their travel instructions precisely. "Now we know" - Charles says - "that from the point of view of the Einsteinville frame of reference both journeys are dynamically symmetrical."

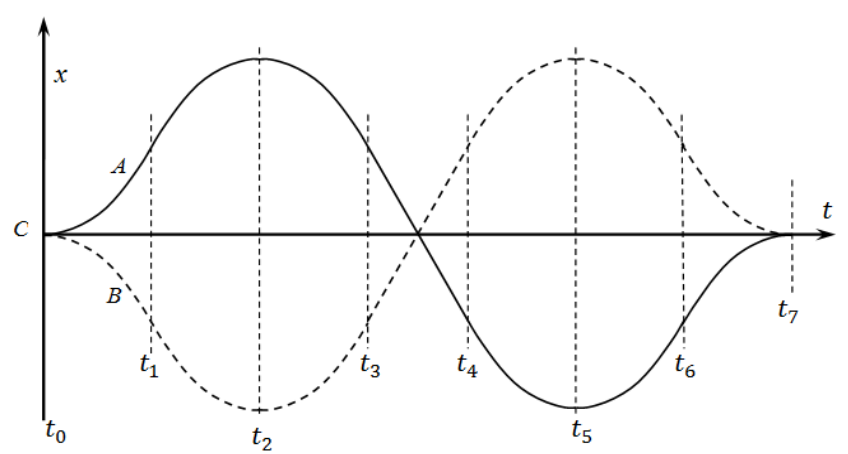

Figure 3. Positions of the moving brothers $A$ and $B$ with respect to $C$ 's proper (stationary) reference frame as determined by integration with time of the acceleration

Albeit the locomotives are uniformly accelerated in their own reference frame, according to SRT they are said not to be uniformly accelerated in the reference frame of the station. In which case the line segments for stages $\left(t_{0}\right.$ to $\left.t_{1}\right)$, $\left(t_{1}\right.$ to $\left.t_{2}\right),\left(t_{2}\right.$ to $\left.t_{3}\right),\left(t_{4}\right.$ to $\left.t_{5}\right),\left(t_{5}\right.$ to $\left.t_{6}\right)$, and $\left(t_{6}\right.$ to $\left.t_{7}\right)$ are not parabolic curves [1, pp. 152-153]. However, at every time point $t$, Alex's locomotive at every position $x(t)$ is strictly symmetric with position $-x(t)$ of Boris's locomotive relative to the Charles's position $x(t) \equiv 0$ on the platform. So the points of maximum displacement from the middle of the platform are reached by both locomotives at the same moment $t_{2}$, and later at $t_{5}$ (figure 3 ).

"Since any asymmetry depending on the direction of the motion - whether to the right or to the left from the platform along $x$ axis - would violate the idea of the homogeneity and isotropy of space," - explains Charles - "you have to admit the following equalities:

$$
t_{r}^{\prime}=t_{r}^{\prime \prime}, t_{b}^{\prime}=t_{b}^{\prime \prime} . "
$$

- "Of course !" - says Alex.

- "So what?" - Boris asks.

- "This is embarrassing for the idea of relativity of simultaneity." - Charles replies - "the uncomfortable truth is that we have a contradiction with arithmetic. It is shown by the following simple transformation starting from inequality (2) and using the equalities (4):

$$
\begin{gathered}
t_{r}^{\prime}-t^{\prime}{ }_{r}<t^{\prime}{ }_{b}-t^{\prime \prime}{ }_{r} \Rightarrow 0-t^{\prime \prime}{ }_{b}<t^{\prime}{ }_{b}-t^{\prime \prime}{ }_{r}-t^{\prime}{ }_{b} \Rightarrow \\
-t^{\prime \prime}{ }_{b}<-t^{\prime \prime}{ }_{r} \Rightarrow t^{\prime \prime}{ }_{r}<t^{\prime \prime}{ }_{b} .
\end{gathered}
$$

Thus, for the last inequality (5) there is an obvious discrepancy with the experimental result from the thought experiment (3). The discrepancy is a consequence of relativity of simultaneity. The choice we face is now clear: either the relativity of simultaneity or the arithmetic is selfcontradictory."

\section{Kinematic Fields and Kinematically Induced Fields}

Maxwell's equations are inconvenient to practical application; this is why, to satisfy engineering demands suitable adjustments were made. Such 'accommodations' are, for example, the expression for EMF

$$
E M F=-\frac{d \Phi}{d t}
$$

where $\Phi$ is a magnetic flux through a closed circuit; and differential equations of the second order for the electric and magnetic vectors,

$$
\boldsymbol{\nabla}^{2} \mathbf{E}-\frac{1}{c^{2}} \frac{\partial^{2} \mathbf{E}}{\partial t^{2}}=\mathbf{0}, \quad \nabla^{2} \mathbf{B}-\frac{1}{c^{2}} \frac{\partial^{2} \mathbf{B}}{\partial t^{2}}=\mathbf{0},
$$

where c is speed of light. So called 'flux rule' (6) gives a direct solution for the differential equation. The wave equations (7) are easier to resolve than system of differential equations of the first order given by Maxwell himself.

An unintended consequence is that the new forms loose the omnipotent quality of the prime description. Consider, for instance, a version of Faraday's disk presented in Figure 4. The conductive disk and a $\Pi$-shaped sliding contact are positioned in a gap between the poles of a permanent magnet (a closing yoke is similar to one shown in Figure 5). When the disk is rotating with a constant angle speed - $\boldsymbol{\omega}$, a voltmeter connecting the disk's centre and the sliding contact shows a non-zero voltage $V$, although no change in the magnetic flux $\Phi$ occurs. It is especially important to note that the same voltage $V$ persists if the disk is stable, but magnet and sliding contact are rotating with the opposite angle speed w.

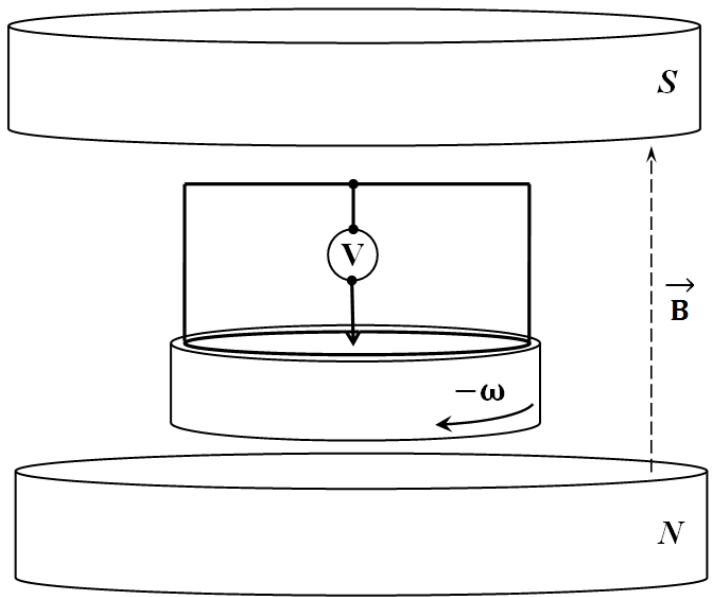

Figure 4. A version of the Faraday disk 
The magneto-kinematic effect - motion of a magnetic field with a moving permanent magnet, creates a kinematically induced electric field. This was first demonstrated for rotary magnetic field motion by N.E. Zajev and V.I. Dokuchajev [6] (magneto-kinematical Zajev-Dokuchajev effect, ZD-effect). This has been confirmed more recently [7, 8] and also established in the case of the rectilinear motion of a permanent magnet [9]. All these experiments proved a natural existence of the 'dragging' the magnetic field $\mathbf{B}$ by a moving at a speed $\mathbf{v}$ permanent magnet, which induces in the laboratory electric field $\mathbf{E}=\mathbf{B} \times \mathbf{v}$ (in the SI units) [10].

Let the gap between poles be relatively small compare to diameter of the magnet (Figure 5). Then the fringe effect is negligible and the field inside the gap is basically uniform. In the rectangular coordinates $(x, y, z)$ the magnetic induction B has components $(0,0, B)$. The magnet is rotating with a constant angular velocity $\boldsymbol{\omega}$. The linear velocity $\mathbf{v}$ has components $(-\omega y, \omega x, 0)$ and the electric tension $\mathbf{E}$ has components $(-B \omega x,-B \omega y, 0)$. Thus we have a stationary in time vector field converging to the axis of rotation and progressively diminishing to the zero-value in the very centre. We deduce from this that $\operatorname{curl} \mathbf{E} \equiv 0$ and $\operatorname{div} \mathbf{E}=-2 B \omega \neq$ 0 without any charges anywhere in the gap, i.e. in this instance the stationary electric field cannot satisfy the Maxwell's equations.

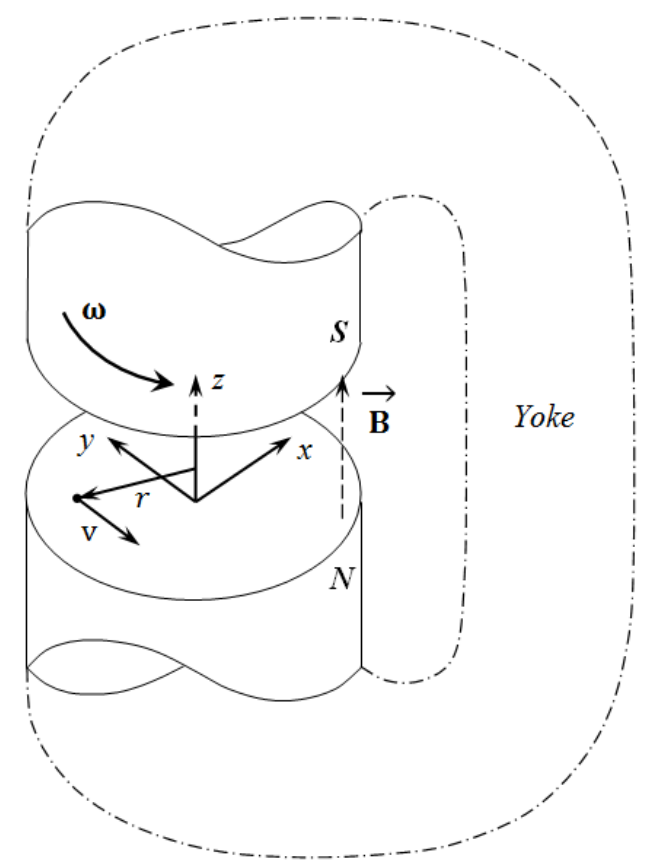

Figure 5. Permanent magnet rotating about axis of cylindrical symmetry

It would be natural to expect the existence in the nature of an electro-kinematical phenomenon, symmetric to the ZDeffect. J. C. Maxwell dreamed and hoped to "at least verify our supposition that a moving electrified body is equivalent to an electric current" [11, p. 370, article 770]. Moreover, he suggested the real scheme of a relevant experimentation, but... "The unified view of electricity and magnetism which was then emerging from Maxwell's work suggested that any moving charge ought to cause a magnetic field, but experimental proof was hard to come by" [12, p. 241]. Indeed, the existence of the magnetic field $\mathbf{B}=\frac{1}{c^{2}}(\mathbf{v} \times \mathbf{E})$ (too small in value) induced in the laboratory by moving at a velocity $\mathrm{v}$ charged (or polarized) rigid bodies, so called convection currents, have been proven in experiments by $\mathrm{H}$. Rowland and A.A. Eihenwald (RE-effect) [13, 14]. These kinematically induced fields also may not meet Maxwell-s equations. For instance, the field of a capacitor with circled plates, rotating about their centres. A new example of such fields is examined in the following section with due regards to special relativity theory (SRT).

\section{Fields of Kinematical Origin Induced by a Moving Magnetized Sphere}

A sphere of diameter $d$, with a uniform magnetization $\mathbf{M}$ of magnitude $M$ and parallel to any axis, is embedded in a non-permeable medium (Figure 6). Let $(r, \theta, \varphi)$ be the system of spherical coordinates with polar axis directed along the vector $M$ and with its origin in the centre of the sphere. The magnetic field in the external space may be expressed through simple elementary functions [15, p.183]. In this case the components of the magnetic flux density (SI - units) are

$$
B_{r}=p \frac{\cos \theta}{r^{3}}, B_{\theta}=\frac{p}{2} \frac{\sin \theta}{r^{3}}, B_{\varphi}=0,
$$

where $p$ is a constant numerical coefficient depending on the system of units used. The projections of the vector B on axis of a Cartesian (rectangular) coordinates are

$$
\begin{gathered}
B_{x}=\frac{3 p}{2} \frac{\sin \theta \cos \theta \cos \varphi}{r^{3}}, B_{y}=\frac{3 p}{2} \frac{\sin \theta \cos \theta \sin \varphi}{r^{3}}, B_{z} \\
=\frac{p}{2} \frac{\left(2-3 \sin ^{2} \theta\right)}{r^{3}} .
\end{gathered}
$$

The length of a radius-vector into an arbitrary point $A$ is $r=\sqrt{x^{2}+y^{2}+z^{2}}$ and occurring trigonometry functions may be expressed as following:

$$
\begin{gathered}
\sin \theta=\frac{\sqrt{x^{2}+y^{2}}}{r}, \cos \theta=\frac{z}{r}, \quad \sin \varphi=\frac{y}{\sqrt{x^{2}+y^{2}}}, \cos \varphi \\
=\frac{x}{\sqrt{x^{2}+y^{2}}} .
\end{gathered}
$$

Substituting these expressions into Cartesian projections of the vector $B$ we have

$$
B_{x}=\frac{3 p}{2} \frac{x z}{r^{5}}, \quad B_{y}=\frac{3 p}{2} \frac{y z}{r^{5}}, \quad B_{z}=\frac{p}{2} \frac{2 z^{2}-x^{2}-y^{2}}{r^{5}} .
$$

Let the frame of reference, where the magnetised sphere is at rest, have the axes $(x, y, z)$ in parallel with the axes $X, Y, Z$ of the laboratory reference frame (Figure 6). Assuming that the sphere is moving relative to the laboratory reference frame along the $Z$ axis at a constant velocity $\mathbf{v}=(0,0, v)$. The magnetised sphere provides a magnetic field with space- 
variable flux density B. The Lorentz transformations[1] gives the following connections between the values in both systems:

$$
\begin{gathered}
x=X, y=Y, \quad z=\gamma(Z-v t) ; B_{X}=\gamma B_{x}, B_{Y}= \\
\gamma B_{y}, B_{Z}=B_{z} .
\end{gathered}
$$

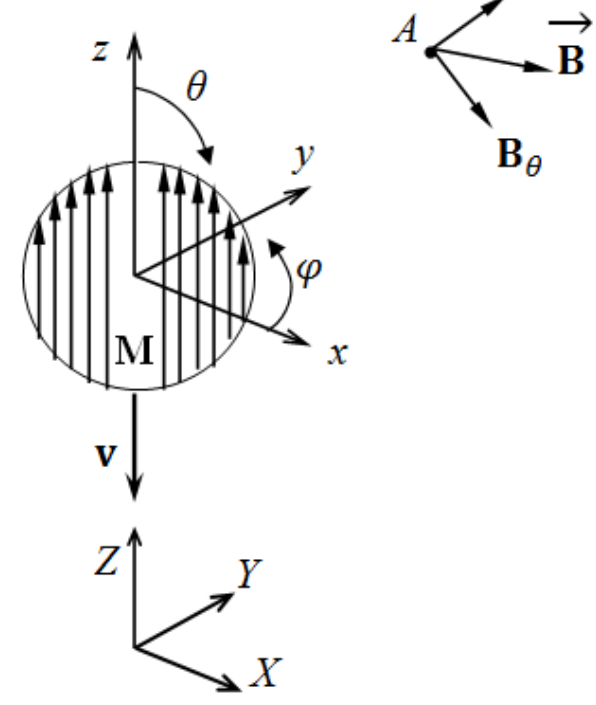

Figure 6. Uniformly magnetized sphere moving along Z-axis

Here the relativistic factor $\gamma=\left(1-v^{2} / c^{2}\right)^{-1 / 2}$, where $c$ is the speed of light. Substituting expressions (8) into equations (9) we obtain components of the magnetic vector $\mathbf{B}$ in any point $(X, Y, Z)$ of the laboratory system corresponding to the point $(x, y, z)$ of the moving one:

$$
\begin{gathered}
\frac{\partial^{2} B_{Z}}{\partial X^{2}}=3 \frac{Y^{4}-4 X^{4}-4 \gamma^{4}(Z-v t)^{4}-3 X^{2} Y^{2}+27 \gamma^{2}(Z-v t)^{2} X^{2}-3 \gamma^{2}(Z-v t)^{2} Y^{2}}{R^{9}} \\
\frac{\partial^{2} B_{Z}}{\partial Y^{2}}=3 \frac{X^{4}-4 Y^{4}-4 \gamma^{4}(Z-v t)^{4}-3 X^{2} Y^{2}-3 \gamma^{2}(Z-v t)^{2} X^{2}+27 \gamma^{2}(Z-v t)^{2} Y^{2}}{R^{9}} ; \\
\frac{\partial^{2} B_{Z}}{\partial Z^{2}}=3 \gamma^{2} \frac{3 X^{4}+3 Y^{4}+8 \gamma^{4}(Z-v t)^{4}+6 X^{2} Y^{2}-24 \gamma^{2}(Z-v t)^{2} X^{2}-24 \gamma^{2}(Z-v t)^{2} Y^{2}}{R^{9}}
\end{gathered}
$$

From above formulae the application of Laplacian operator to $B_{Z}$ gives

$$
\nabla^{2} B_{Z}=\frac{\partial^{2} B_{Z}}{\partial X^{2}}+\frac{\partial^{2} B_{Z}}{\partial Y^{2}}+\frac{\partial^{2} B_{Z}}{\partial Z^{2}}=3\left(\gamma^{2}-1\right) \frac{3 X^{4}+3 Y^{4}+8 \gamma^{4}(Z-v t)^{4}+6 X^{2} Y^{2}-24 \gamma^{2}(Z-v t)^{2} X^{2}-24 \gamma^{2}(Z-v t)^{2} Y^{2}}{R^{9}}
$$

The partial derivative over time is

$$
\frac{\partial^{2} B Z}{\partial t^{2}}=3 \gamma^{2} v^{2} \frac{3 X^{4}+3 Y^{4}+8 \gamma^{4}(Z-v t)^{4}+6 X^{2} Y^{2}-24 \gamma^{2}(Z-v t)^{2} X^{2}-24 \gamma^{2}(Z-v t)^{2} Y^{2}}{R^{9}} .
$$

For comparison (12) and (13), it is necessary to recalculate the expression $\left(\gamma^{2}-1\right)$ :

$$
\gamma^{2}-1=\frac{1}{1-v^{2} / c^{2}}-1=\frac{1-1+v^{2} / c^{2}}{1-v^{2} / c^{2}}=\frac{v^{2} / c^{2}}{1-v^{2} / c^{2}}=\gamma^{2} \frac{v^{2}}{c^{2}}
$$

Now it is evident that $\nabla^{2} B_{Z}=\frac{\partial^{2} B_{Z}}{\partial t^{2}} / c^{2}$, and consequently we get the wave equation for the magnetic Z-component $B_{Z}$ :

$$
\nabla^{2} B_{Z}-\frac{1}{c^{2}} \frac{\partial^{2} B_{Z}}{\partial t^{2}}=0
$$

The partial derivatives of the magnetic component $B_{X}(10)$ in the laboratory system are:

$$
\frac{\partial B_{X}}{\partial X}=3 \gamma^{2}(Z-v t) \frac{Y^{2}-4 X+\gamma^{2}(Z-v t)^{2}}{R^{7}}
$$




$$
\begin{gathered}
\frac{\partial B_{X}}{\partial Y}=-15 \gamma^{2} \frac{X Y(Z-v t)}{R^{7}} \\
\frac{\partial B_{X}}{\partial Z}=3 \gamma^{2} X \frac{X^{2}+Y^{2}-4 \gamma^{2}(Z-v t)^{2}}{R^{7}} \\
\frac{\partial B_{X}}{\partial t}=3 v \gamma^{2} X \frac{4 \gamma^{2}(Z-v t)^{2}-X^{2}-Y^{2}}{R^{7}} .
\end{gathered}
$$

Let us find all second partial derivatives of the magnetic $\mathrm{X}$ component $B_{X}$ :

$$
\begin{aligned}
& \frac{\partial^{2} B_{X}}{\partial X^{2}}=15 \gamma^{2} X(Z-v t) \frac{4 X^{2}-3 Y^{2}-3 \gamma^{2}(X-v t)^{2}}{R^{9}} \\
& \frac{\partial^{2} B_{X}}{\partial Y^{2}}=-15 \gamma^{2} X(Z-v t) \frac{X^{2}-6 Y^{2}+\gamma^{2}(Z-v t)^{2}}{R^{9}} \\
& \frac{\partial^{2} B_{X}}{\partial Z^{2}}=-15 \gamma^{4} X(Z-v t) \frac{3 X^{2}+3 Y^{2}-4 \gamma^{2}(Z-v t)^{2}}{R^{9}}
\end{aligned}
$$

Application of the Laplacian operator to the magnetic component $B_{X}$ gives the expression:

$$
\nabla^{2} B_{X}=15 \gamma^{2} X(Z-v t) \frac{3 X^{2}+3 Y^{2}-4 \gamma^{2}(Z-v t)^{2}}{R^{9}}\left(1-\gamma^{2}\right),
$$

where from, taking into account (14), we obtain

$$
\nabla^{2} B_{X}=-15 \gamma^{4} v^{2} X(Z-v t) \frac{3 X^{2}+3 Y^{2}-4 \gamma^{2}(Z-v t)^{2}}{R^{9}} \frac{1}{c^{2}} .
$$

But then, for the derivative over time we have

$$
\frac{\partial^{2} B_{X}}{\partial t^{2}}=\frac{\partial}{\partial t}\left(\frac{\partial B_{X}}{\partial t}\right)=-15 \gamma^{4} v^{2} X(Z-v t) \frac{3 X^{2}+3 Y^{2}-4 \gamma^{2}(Z-v t)^{2}}{R^{9}} .
$$

From comparison (16) and (17) therefore, it follows that wave equation for the magnetic $\mathrm{X}$-component is valid:

$$
\nabla^{2} B_{X}-\frac{1}{c^{2}} \frac{\partial^{2} B_{X}}{\partial t^{2}}=0
$$

By virtue of cylindrical symmetry the same equality takes place for the magnetic Y-component $B_{Y}(10)$ :

$$
\nabla^{2} B_{Y}-\frac{1}{c^{2}} \frac{\partial^{2} B_{Y}}{\partial t^{2}}=0
$$

So, just the same result turns out for the full magnetic vector:

$$
\boldsymbol{\nabla}^{2} \mathbf{B}-\frac{1}{c^{2}} \frac{\partial^{2} \mathbf{B}}{\partial t^{2}}=\mathbf{0}
$$

As soon as the corresponding electric field $E=B \times v$ of kinematical origin has the components proportional to that of magnetic vector (11), a similar wave equation

$$
\nabla^{2} \mathbf{E}-\frac{1}{c^{2}} \frac{\partial^{2} \mathbf{E}}{\partial t^{2}}=\mathbf{0}
$$

is valid for the electric vector.

In contrast to the above mentioned examples, here is, so to say, an over-exaggerated compliance to the wave equation. It is obvious that the field is moving with the velocity $\mathrm{v}=(0$, $0, v)$, which is characteristic for the field carrier. So, it would be rather natural to expect the equations

$$
\boldsymbol{\nabla}^{2} \mathbf{E}-\frac{1}{v^{2}} \frac{\partial^{2} \mathbf{E}}{\partial t^{2}}=\mathbf{0}, \boldsymbol{\nabla}^{2} \mathbf{B}-\frac{1}{v^{2}} \frac{\partial^{2} \mathbf{B}}{\partial t^{2}}=\mathbf{0}
$$

to be held instead of the equations (18) and (19), but it isn't at all. As soon as the equations (20) are not valid, the only conclusion is that a kinematic field does not propagate in space like a wave $[10,16,17]$. It should be noted that nonrelativistic approach does confirm the equations (20) to be invalid [18] about kinematic fields.

\section{Fields of a Moving Point Charge}

Let us remember how the field of a moving point charge had been derived. "According the formulas for retarded potentials, the field at the point of observation $P(x, y, z)$ at time $t$ is determined by the state of motion of the charge at the earlier time $t^{\prime}$, for which the time of propagation of the light signal from the point $\mathrm{r}_{\mathrm{e}}\left(t^{\prime}\right)$, where the charge was located, to the field point $P$ just coincides with the difference $t-t^{\prime}$. Let $\mathrm{R}(t)=\mathrm{r}-\mathrm{r}_{\mathrm{e}}\left(t^{\prime}\right)$ be the radius vector from the charge $e$ to the point $P$; like $\mathrm{r}_{\mathrm{e}}(t)$ it is a given function of the time (Figure 7). Then the time $t^{\prime}$ is determined by the equation $t^{\prime}+R(t) / c=t$. For each value of $t$ this equation has just one root. ... Now once more transforming to threedimensional notations, we obtain, for the potentials of the field produced by an arbitrary moving point charge, the following expressions:

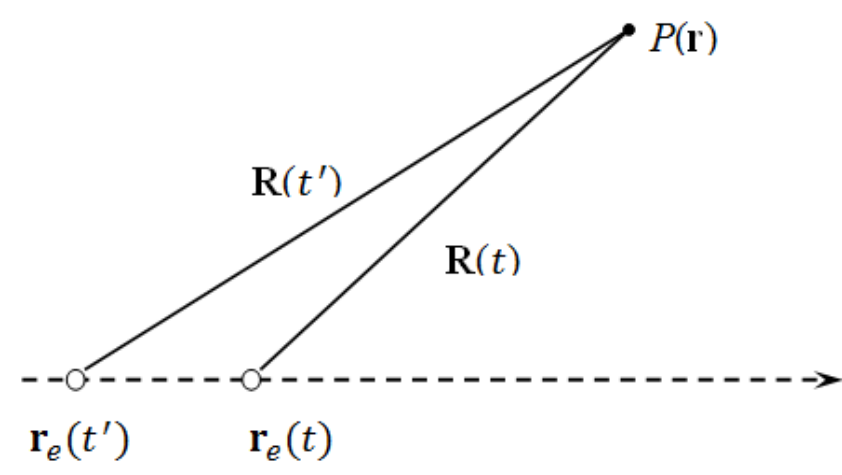

Figure 7. Arbitrary moving point charge

$$
\varphi=\frac{e}{\left(R-\frac{\mathbf{v} \cdot \mathbf{R}}{c}\right)}, \quad \mathbf{A}=\frac{e \mathbf{v}}{c\left(R-\frac{\mathbf{v} \cdot \mathbf{R}}{c}\right)},
$$

where $\mathbf{R}$ is the radius vector, taken from the point where the charge is located to the point of observation $P$, and all the quantities on the right sides of the equations must be evaluated at the time $t^{\prime}$ determined from the previous equation. The potentials of the field, in the form $(*)$, are called the Lienard-Wiechert potentials." [19, p. 174]

What such reasoning seems to forget is an implicit assumption, for the field, of a permanent flowing out from its source. Indeed, so long as the potential propagates with the same light-speed " $c$ " regardless of what sort of movement the charge performs, this travel remains in the case of a uniform motion of the charge at a constant velocity $\mathbf{v}$. Since the light 
speed does not depend on $\mathbf{v}$, the travel of potential persists when $\mathbf{v}$ tends to zero. Thus we should accept that an incessant outward flow of potential from a charged source always exists in every static field. This proposition must be explicitly added to the axiomatic basis, when SRT is extended over electricity and magnetism.

This way of thinking logically leads to the conclusion about the behaviour of the field itself. The authors use the Lienard-Wiechert potentials $(*)$ to obtain electric and magnetic vectors. "With the aid of these formulas, there is no difficulty in carrying out the calculation of the fields $\mathbf{E}$ and $\mathbf{H}$. Omitting the intermediate calculations, we give the final results:

$$
\begin{gathered}
\mathbf{E}=e \frac{1-v^{2} / c^{2}}{\left(R-\frac{\mathbf{R} \cdot \mathbf{v}}{c}\right)^{3}}\left(\mathbf{R}-\frac{\mathbf{v}}{c} R\right)+\frac{e}{c^{2}\left(R-\frac{\mathbf{R} \cdot \mathbf{v}}{c}\right)^{3}} \mathbf{R} \times\left\{\left(\mathbf{R}-\frac{\mathbf{v}}{c} R\right) \times \dot{\mathbf{v}}\right\}, \\
\mathbf{H}=\frac{1}{R} \mathbf{R} \times \mathbf{E} .
\end{gathered}
$$

Here $\dot{\mathbf{v}}=\partial \mathbf{v} / \partial t^{\prime} ;$ all quantities on the right sides of the equations refer to the time $t^{\prime}$. It is interesting to note that the magnetic field turns out to be everywhere perpendicular to the electric. The electric field (**) consists of two parts of different type. The first term depends only on the velocity of the particle (and not on its acceleration) and varies at large distances like $1 / R^{2}$. The second term depends on the acceleration, and for large $R$ it varies like $1 / R . "[19, p .175]$

The relativity theory states a total equivalence between an uniformly accelerated frame of reference $K_{a}$ and inertial frame $K_{\mathrm{g}}$ having uniform gravitational field, so that $\mathbf{g}=-\boldsymbol{a}$. "A point charge, unfixed in $K_{g}$, moves in free fall at the acceleration $\boldsymbol{a}=\mathbf{g}$. According to $(* *)$ and $(* * *)$ it is emitting electromagnetic waves. The charge $e$, stationary relative to some inertial frame $K$, is not accelerated and, as it seems at first glance, it should not emit in the frame $K_{a}$. Nevertheless, in actuality this charge emits in $K_{a}$ just as it does in the frame $K_{g}$, i.e. the equivalence principle is undeniably obeyed." [20]

This quotation gives the following thought-provoking idea. Let us consider an observer $O_{1}$ moving rectilinearly with a constant acceleration $\boldsymbol{a}_{1}$ directed to the charge $e$. If V.L. Ginzburg is right, the observer $O_{1}$ perceives radiation emitted by $e$ in accordance with the equations $(* *)$ and $(* * *)$. Another observer $O_{2}$ moving to the charge $e$ with a constant acceleration $\boldsymbol{a}_{2} \neq \boldsymbol{a}_{1}$, in turn perceives radiation sourced from $e$. A third observer $O_{3}$ moving with acceleration $\boldsymbol{a}_{3} \neq \boldsymbol{a}_{2} \neq \boldsymbol{a}_{1}$, is also irradiated by the charge $e$, and so on... Using this line of reasoning, we must admit the existence of a permanent stream of radiation, with unlimited intensity, flowing into surrounding space outward from the charge $e$, being at rest in the inertial frame $K$.

V.L. Ginzburg in [20] appeals to the well-known decomposition of field by German Helmholtz into longitudinal and transverse components and believes this radiation to be non-photonic because in either inertial frame $K$ the charge $e$ has a constant velocity $\mathbf{v}$. But first of all, the field of such a charge does obey the relativistic wave equations similar to (18) and (19) owing to the implicit axiom. Secondly, for an observer in every $K_{a}$ the value $\dot{\mathbf{v}} \neq \mathbf{0}$, therefore the last part of radiation in $(* *)$ cannot be neglected. Moreover, thirdly, Ginzburg accepts that "in free fall motion a charge emits EM-waves", i.e. actually simple photons. Thus, the radiation of charge $e$ in a uniformly accelerated frame $K_{a}$ should be obligatory photonic in full agreement with the equivalence principle, which is vehemently up-held by Ginzburg himself. (It should also be noted that the hypothetical "Fulling-Davies-Unruh effect" has nothing to do with the considered problem. The so called "black-body Unruh radiation" does not require any charge as a source to be emitted.)

\section{Conclusion}

The logical analysis of the Triplet paradox leads to an irreconcilable contradiction between the thought experiment result and the arithmetic. This is not to say that SRT is wrong, but it is important to have a scientific explanation for the above inconsistency. It is very likely that some boundary of the validated region is trespassed, in which case the relativity of simultaneity must be reviewed because here SRT does not hold the test. It is common knowledge that Maxwell's laws have required no changes at high velocities, and remain accurately correct, even when $\mathrm{v}$ approaches close to the speed of light. However, as was shown in the above text, sometimes such "invariance" gives rise to the loosing of sense. Even worse is the appearance of a vicious circle with the equivalence principle applied to electromagnetism. So, the unsolved contradiction of SRT leads to a dramatic discrepancy in the realm of general relativity theory.

Sometimes in the past, a doubt occurred, which belied the conventional certainty in full compatibility between SRT and classical electrodynamics. For instance, Oleg D. Jefimenko pointed out that it would be highly consistent to implement the Fitzgerald's contraction not only to a rigid body, but also to the density of a charge distributed in space, while the invariance of Maxwell's equations is under consideration. "Einstein (as well as Lorentz, Larmor, and Poincaré, who preceded Einstiein in development and use of relativistic transformations and in the demonstration of the invariance of Maxwell's equations under these transformations) used prerelativistic Maxwell's equations, in which the charge density was meant to be the electrostatic charge density" [21]. Unfortunately, this highly important observation is neglected, as before, by the scientific community. If any theory comes to terms with reality, the sharp bordering line around its validated region must be outlined.

The study of electromagnetism requires now a new strategy attuned to the fact that any extension of SRT on electromagnetic phenomena is fraught with serious consequences associated with a probable departure from the validated domain. God knows what unexpected tricks a venturesome implementation of the theory might manifest? So, any attempt to sound out a possible demarcation line would be in the vested interest for physical science. A crucial solution could be the suggested in [22] experimental 
checking in order to examine the field configuration of an electron beam provided by a linear or circling accelerator. The electric lines of force could have some another map. Such a possibility must not be ruled out beforehand.

\section{Acknowledgement}

The author sincerely thanks Mr Michael Kyle for the indispensable help while preparing this paper.

\section{References}

[1] A.P. French, Special Relativity. Thomas Nelson \& Sons, Great Britain, 1981.

[2] S. Boblest, T. Müller and G. Wunner, "Twin paradox in de Sitter space-time”, Eur. J. Phys, № 32, p. 1117, 2011.

[3] H. Minkowski, "Raum und Zeit", Physikalische Zeitschrift, № 10, p. 75, 1908.

[4] C. Ranzan, "Einstein's Simple Mathematical Trick and the Illusion of a Constant Speed of Light", Applied Physics Research, vol. 5, № 4, pp. 85-95, 2013.

[5] Leus V.A. "Triplet paradox in special relativity", Open Journal of Modern Physics, vol.2, №1, pp.11-15, 2015.

[6] Zajev N.E. and Dokuchajev V.I., Electrotechnika (Electrical Engineering, in Russian) 11, 64 (1964).

[7] Leus V.A. and Zatolokin V.N. "The magneto-kinematical effect”. IJEEE 43 (4), 245 (2006).

[8] Leus V. and Taylor S., "On the motion of the field of a permanent magnet”, Eur. J. Phys., Vol. 32, No 5, 1179-1192, (2011).

[9] Taylor, S., and Leus, V. A. "The magneto-kinematic effect for the case of rectilinear motion". Eur.J.Phys., Vol. 33, No 4, 837$852,(2012)$.

[10] Leus V. and Taylor S.., "Experimental Evidence for the Magneto-kinematic Effect", PIERS Proceedings, (Moscow), pp.1040-1048 (August 2012).
[11] Maxwell J C 1873 A Treatise on Electricity and Magnetism, Vol 2 (Oxford: Clarendon Press)

[12] Purcell E M 1984 Electricity and Magnetism vol $22^{\text {nd }}$ edn (New York: McGraw-Hill)

[13] Rowland H., "On the Magnetic Effect of Electric Convection", American Journal of Science, Vol. XV, No. 3, 30-33, (1878).

[14] Eihenwald A. A., "Izbrannye raboty - Selected works," Physico-matematicheskaja literatura, Moscow, (in Russian), (1956)

[15] Jackson J. D., "Classical Electrodynamics" (3 ${ }^{\text {th }}$ Ed), John Wiley \& Sons, New York, (1999).

[16] Leus VA, Smith RT, Maher S. "The Physical Entity of Vector Potential in Electromagnetism". Applied Physics Research. 2013; vol. 5 (4): 56-68.

[17] Leus VA, Smith RT, Maher S. "Two Types of Electromagnetic Induction Existing in Nature", PIER Symposium, Stockholm Sweden, August 12-15, 2013.

[18] Leus, V.A. "The magneto-kinematical and electro-kinematical fields". Progress In Electromagnetics Research M, Vol. 32, 27-41, (2013).

[19] L.D. Landau and E.M. Lifshitz. "The Classical Theory of Fields - Course of theoretical physics". Vol. 2. Pergamon Press. Oxford, London, New York, Paris (1960).

[20] Ginzburg V.L. "On emission and the force of radiating friction during uniformly accelerated motion of a charge". Uspekhi Fizicheskih Nauk (Advance in Physics). Vol. 98, № 3, 569585, 1969, (in Russian).

[21] Oleg D. Jefimenko, "On the Relativistic Invariance of Maxwell's Equations”, Z. Naturforsch, 54a, pp. 637-644 (1999).

[22] V.A. Leus "On the Field of a Moving Charge", Open Journal of Modern Physics, vol.1, №1, pp. 1-7, 2014. 